Pacific

Journal of

Mathematics

\title{
INTERIOR GRADIENT ESTIMATES \\ FOR ANISOTROPIC MEAN-CURVATURE FLOW
}

Julie Clutterbuck

Volume $229 \quad$ No. 1

January 2007 


\title{
INTERIOR GRADIENT ESTIMATES FOR ANISOTROPIC MEAN-CURVATURE FLOW
}

\author{
JULie ClutterbuCK
}

\begin{abstract}
In this paper we consider the evolution of a graph-like hypersurface by anisotropic mean-curvature flow, under some restrictions on the anisotropic area integrand. We find interior estimates (in both time and space) on the gradient of such hypersurfaces, depending only on the height of the graph and the anisotropic area integrand.
\end{abstract}

\section{Introduction}

Consider the evolution of a hypersurface by its mean curvature,

$$
\frac{d}{d t} \boldsymbol{x}(y, t)=\boldsymbol{H}(y, t), \quad y \in \boldsymbol{M},
$$

where $\boldsymbol{x}: \boldsymbol{M}^{n} \times[0, T] \rightarrow \mathbb{R}^{n+1}$ is the immersion of a manifold $\boldsymbol{M}$ at each time $t$, and $\boldsymbol{H}$ is the mean curvature vector. The hypersurface $\boldsymbol{M}$ can be written as a graph when a fixed vector $\omega \in \mathbb{R}^{n+1}$ can be found so that, for a choice of unit normal $v$, we have $\langle v, \omega\rangle>0$ everywhere. Given the image $\boldsymbol{x}(y, t)$ of a point $y \in \boldsymbol{M}$, the height of $\boldsymbol{M}$ above the hyperplane defined by $\omega$ is denoted by $u=\langle\boldsymbol{x}, \omega\rangle$, and the gradient function is given by

$$
v=\langle v, \omega\rangle^{-1}=\sqrt{1+|D u|^{2}} .
$$

Ecker and Huisken [1989] established that, when initial data is given by an entire Lipschitz graph with a linear-growth bound, there is a smooth solution to (1) for all times. An important step in this proof was showing that the solutions remain graphs; this was done by showing that $v$ is bounded above, with a constant depending on the initial Lipschitz bound. In [Ecker and Huisken 1991], it was established that the Lipschitz bound need only be local; our estimates are intended in the spirit of the local gradient estimates of Section 2 of this latter paper.

Such gradient estimates may be found even if the initial data is not Lipschitz. Evans and Spruck [1992] showed that, under mean-curvature flow, surfaces that may initially be written locally as a continuous graph become smooth for $t>0$.

MSC2000: 35K55, 53A07.

Keywords: evolution equations, anisotropic mean curvature flow, gradient estimates. 
The level-set method was also used by Barles, Biton and Ley [Barles et al. 2002] to find similar gradient estimates for a more general class of equations. More recently, Colding and Minicozzi [2004] found an explicit local estimate in the form

$$
|D u(x, t)| \leq \exp \left(c\left(1+t^{-1 / 2}\|u\|_{\infty}\right)^{2}\right)
$$

for solutions over a ball $B_{R}(x)$ with $R \geq C \sqrt{t}$. Here, the constant $c$ depends only on the dimension. This estimate does not depend on an initial gradient estimate.

We find an analogous result for anisotropic mean-curvature flow, under some restrictions on the anisotropy. Such estimates are an important step in finding existence results for a variety of boundary-value problems with non-smooth initial data, as in [Andrews and Clutterbuck 2005].

We will follow the exposition in [Andrews 2001], in particular Section 8, in which the evolution equation for graph-like surfaces is derived.

Consider surfaces $\boldsymbol{M}$ with local embeddings

$$
x=y^{i} e_{i}+u\left(y^{1}, \ldots, y^{n}\right) e_{0},
$$

and normal

$$
v=D u-\phi^{0}=\sum_{i=1}^{n} u_{i} \phi^{i}-\phi^{0},
$$

where $\left\{\phi^{0}, \phi^{1}, \ldots, \phi^{n}\right\}$ and $\left\{e_{0}, e_{1}, \ldots, e_{n}\right\}$ are dual bases for the cotangent space $V^{*} \cong \mathbb{R}^{n+1}$ and tangent space $V \cong \mathbb{R}^{n+1}$, respectively.

The anisotropic mean-curvature flow for such a surface is given by

$$
u_{t}=\left.F D^{2} F\right|_{D u-\phi^{0}}\left(\phi^{i}, \phi^{j}\right) D^{2} u\left(e_{i}, e_{j}\right)
$$

(the homogeneous degree-zero mobility function $m=m(v)$ of [Andrews 2001] is here taken to be identically 1 ). The anisotropic area integrand $F: V^{*} \rightarrow \mathbb{R}$ is a positive, convex function that is $C^{3}$ on $V^{*} \backslash\{0\}$, and homogeneous of degree one, so that $F(\lambda v)=\lambda F(v)$ for all $v \in V^{*}$ and scalars $\lambda>0$. The level sets of $F$ are denoted by

$$
\Sigma_{\lambda}:=\left\{v \in V^{*}: F(v)=\lambda\right\} .
$$

We impose the condition that the convex hull of each level set, $\left\{v \in V^{*}: F(v) \leq \lambda\right\}$, must be uniformly convex.

Example. In the isotropic case, we have $F(v)^{2}=\sum_{i=0}^{n}\left(v_{i}\right)^{2}$, and the level sets of $F$ are spheres. The coefficients are those of mean curvature:

$$
\left.F D^{2} F\right|_{D u-\phi^{0}}\left(\phi^{i}, \phi^{j}\right)=\delta_{i j}-u_{i} u_{j}\left(1+|D u|^{2}\right)^{-1} .
$$

We define two further conditions on $F$ :

Smallness of third derivatives condition. Set

$$
Q_{\nu}(p, q, r):=\left.F^{2}(v) D^{3} F\right|_{\nu}(p, q, r) .
$$


The condition is satisfied when

$$
Q_{\nu}(p, q, r) \leq C_{1}\left(\left.\left.\left.F(v)^{3} D^{2} F\right|_{\nu}(p, p) D^{2} F\right|_{\nu}(q, q) D^{2} F\right|_{\nu}(r, r)\right)^{1 / 2}
$$

for all $p, q, r$ tangent to the level set $\Sigma_{F(v)}$ at $v$. Here, $C_{1}$ is a positive constant dependent on $n$.

This $Q$ is the Cartan tensor of Bao, Chern and Shen [Bao et al. 2000], or the tensor $Q$ of [Andrews 2001] restricted to the tangent space of the level set. The restriction here excludes anisotropic area integrands that deviate too far from the isotropic case (such as approximations to the crystalline case). Similar restrictions on the third derivatives of $F$ are made in studies of the elliptic problem (see, for example, [Winklmann 2005]).

Symmetry condition. This is satisfied when

$$
F\left(p+\phi^{0}\right)=F\left(p-\phi^{0}\right) \quad \text { for all } p=\sum_{i=1}^{n} p_{i} \phi^{i} .
$$

Example. The isotropic case clearly satisfies the second condition, as well as the first one with $C_{1}=0$.

We impose these conditions singly — to find two time-interior gradient estimates for periodic solutions - and jointly — to find a gradient estimate interior in both space and time. In a forthcoming paper with Ben Andrews, such estimates are found for periodic flows without imposing either condition.

Let $u: \mathbb{R}^{n} \times[0, T] \rightarrow \mathbb{R}$ be a $C^{3}$, bounded as $|u(x, t)| \leq M$, solution to the anisotropic mean curvature flow equation (2).

Theorem 1. Let $u$ be periodic, so that $u(x, t)=u(x+L, t)$ for some lattice $L \subset \mathbb{R}^{n}$. If $F$ satisfies condition (3) on smallness of third derivatives with $C_{1}{ }^{2}<4 n^{-1 / 2}$, then

$$
F\left(D u-\phi^{0}\right) \leq \max \left\{t^{q / 2} \exp \left(\frac{A q(|u|-2 M)^{2}}{4 t}\right), P\right\}
$$

for $0<t \leq T^{\prime}$, where $T^{\prime}$ depends on $M$, and $A, P$ and $q>1$ depend on $F$.

Theorem 2. Let $u$ be periodic, so that $u(x, t)=u(x+L, t)$ for some lattice $L \subset \mathbb{R}^{n}$. If $F$ satisfies the symmetry condition (4), then

$$
F\left(D u-\phi^{0}\right) \leq \max \left\{t \exp \left(\frac{A(|u|-2 M)^{2}}{2 t}\right), S\right\}
$$

for $0<t \leq T^{\prime}$, where $T^{\prime}$ depends on $M$, while A depends on $F$, and $S$ on $F$ and $n$.

Theorem 3 (Interior estimate for anisotropic mean curvature flow). Let $n>1$. If $F$ satisfies both condition (3) on smallness of third derivatives with $C_{1}^{2}<2 / \sqrt{n}$, and 
the symmetry condition (4), then

$$
F\left(D u-\phi^{0}\right) \leq \max \left\{t^{q / 2} \exp \left(\frac{A q(|u|-2 M)^{2}}{4 t}\right)\left(R^{2}-2 k t-|x|^{2}\right)^{-r}, P\right\}
$$

for $0<t \leq T^{\prime}$. Here, $A, P$ and $k$ depend only on $F$, while $T^{\prime}>0, q>1$ and $r>1$ depend on $F$ and $M$.

In Section 2 we derive some technical results on $F$ and its derivatives, while in Section 3 we prove Theorems 1 and 2. In the final Section 4 we prove Theorem 3.

This work was part of my Ph.D. thesis, written under the supervision of Dr. Ben Andrews at the Australian National University. I would like to thank him for many interesting discussions and helpful suggestions.

\section{Some results regarding the function $F$}

Uniform convexity implies that $\left.F D^{2} F\right|_{\nu}$ is positive definite on the tangent space of the level set $\Sigma_{F(v)}$. The homogeneity of $F$ leads to the disappearance of some derivatives of $F$ in radial directions:

$$
\begin{aligned}
& \left.D F\right|_{\nu}(v)=F(v), \\
& \left.D^{2} F\right|_{\nu}(v, \cdot)=\left.D^{2} F\right|_{\nu}(\cdot, v)=0, \\
& \left.D\left(F D^{2} F\right)\right|_{v}(v, \cdot, \cdot)=0 .
\end{aligned}
$$

These properties make it more convenient to work not in the space $(T \boldsymbol{M})^{*}$, but rather in the tangent space to the level set $\Sigma_{F(v)}$. Given $v$ normal to $\boldsymbol{M}$ at $x$, we can map $v \in V^{*}$ to $T_{\nu} \Sigma_{F(v)}$ by setting

$$
\hat{v}:=v-r(v) v .
$$

The normal is not in the tangent space itself, as $\left.D F\right|_{\nu}(v) \neq 0$ by (5a). By choosing $r(v)$ appropriately, $\hat{v}$ will be in the tangent space, with

$$
0=\left.D F\right|_{\nu}(\hat{v})=\left.D F\right|_{\nu}(v-r(v) v)=\left.D F\right|_{\nu}(v)-r(v) F(v),
$$

where in the last step we have used (5a). With $r(v)=\left.D F\right|_{v}(v) / F(v)$, we then have

$$
\hat{v}=v-\frac{\left.D F\right|_{v}(v)}{F(v)} v
$$

which is nonzero if $v$ is not parallel to $v$. Consequently, $(5 b)$ implies that, for nonzero $v \in(T \boldsymbol{M})^{*}$,

$$
\left.F D^{2} F\right|_{\nu}(v, v)=\left.F D^{2} F\right|_{\nu}(\hat{v}, \hat{v})>0 .
$$

We consider this as a new metric on $(T \boldsymbol{M})^{*}$, and write

$$
G_{v}(v, w):=\left.F D^{2} F\right|_{v}(v, w) .
$$


Lemma 4. For all $P_{1} \geq 0$,

$$
F\left(p-\phi^{0}\right) \geq P_{1}+F\left(-\phi^{0}\right) \quad \text { implies } \quad F(p) \geq P_{1} .
$$

Proof. It is a simple consequence of convexity, as $F\left(p-\phi^{0}\right) \leq F(p)+F\left(-\phi^{0}\right)$.

Lemma 5. Let $\left\{\phi^{0}, \ldots, \phi^{n}\right\}$ be a basis for $V^{*}$. For each $P>F\left(-\phi^{0}\right)$, there exists $A_{P}>0$ such that

$$
\left.F D^{2} F\right|_{p-\phi^{0}}(p, p) \geq A_{P}
$$

for all $p=\sum_{i=1}^{n} p_{i} \phi^{i}$ with $F\left(p-\phi^{0}\right) \geq P$.

Proof. Set $P_{1}=P-F\left(-\phi^{0}\right)>0$. By Lemma 4, if $F\left(p-\phi^{0}\right) \geq P$, then $F(p) \geq P_{1}$.

Define $B(p):=\left.F D^{2} F\right|_{p-\phi^{0}}(p, p)$. Consider this for a fixed member of the level set $p \in \Sigma_{P_{1}} \cap \operatorname{span}\left\{\phi^{1}, \ldots, \phi^{n}\right\}$; as $p$ is not parallel to $p-\phi^{0}, B(p)$ is positive. Also,

$$
\begin{aligned}
\lim _{s \rightarrow \infty} B(s p) & =\left.\lim _{s \rightarrow 0} F D^{2} F\right|_{s p-\phi^{0}}(s p, s p) \\
& =\left.\lim _{s \rightarrow \infty} F D^{2} F\right|_{s p-\phi^{0}}\left(s p-\left(s p-\phi^{0}\right), s p-\left(s p-\phi^{0}\right)\right) \\
& =\left.\lim _{s \rightarrow \infty} F D^{2} F\right|_{p-\phi^{0} / s}\left(\phi^{0}, \phi^{0}\right)=\left.F D^{2} F\right|_{p}\left(\phi^{0}, \phi^{0}\right)>0,
\end{aligned}
$$

where in the second line the additional terms added vanish according to (5b), while in the third line the scaling in $s$ is allowed as $F D^{2} F$ is homogeneous of degree zero. The final inequality is because $\phi^{0}$ is not parallel to $p$. Since $B(s p)>0$ for $1 \leq s<\infty$, it follows that $\inf _{s \in[1, \infty)} B(s p)=A_{p}>0$, and taking the minimum over all $p$ in the (compact and closed) level set gives

$$
\inf _{\Sigma_{P_{1}} \cap \operatorname{span}\left\{\phi^{1}, \ldots, \phi^{n}\right\}} A_{p}=: A_{P}>0 .
$$

Lemma 6. If $F$ satisfies the symmetry condition (4), then homogeneity implies that

$$
\begin{array}{ll}
\left.D F\right|_{p}\left(\phi^{0}\right)=0, & \left.D^{2} F\right|_{p}\left(\phi^{0}, \phi^{j}\right)=0, \\
\left.D^{3} F\right|_{p}\left(\phi^{0}, \phi^{j}, \phi^{k}\right)=0, & \left.D^{3} F\right|_{p}\left(\phi^{0}, \phi^{0}, \phi^{0}\right)=0,
\end{array}
$$

for all $p=\sum_{i=1}^{n} p_{i} \phi^{i}$ and all $j, k \neq 0$.

Proof. The symmetry condition implies that

$$
\left.D F\right|_{p}\left(\phi^{0}\right)=\lim _{s \rightarrow 0} \frac{1}{s}\left(F\left(p+s \phi^{0} / 2\right)-F\left(p-s \phi^{0} / 2\right)\right)=0 .
$$

The others may be proved similarly.

In the next lemma, we show that the symmetry condition (4) can be used in a similar way to condition (3) on smallness of third derivatives. We will use this in the proof of Theorem 2 . 
Lemma 7. Suppose the symmetry condition (4) holds. For all $\varepsilon>0$, we can find $S_{\varepsilon}$ such that, if $p=\sum_{i=1}^{n} p_{i} \phi^{i}$ satisfies $F\left(p-\phi^{0}\right) \geq S_{\varepsilon}$, then

$$
\left|F D\left(F D^{2} F\right)\right|_{p-\phi^{0}}(p, \hat{q}, \hat{q})\left|\leq \varepsilon\left(\left.F D^{2} F\right|_{p-\phi^{0}}(p, p)\right)^{1 / 2} F D^{2} F\right|_{p-\phi^{0}}(q, q)
$$

for all $q=\sum_{i=1}^{n} q_{i} \phi^{i}$.

Proof. Let $\varepsilon>0$ be given. As (7) is unchanged under the mapping $q \mapsto s q$, we consider only those $q$ on a fixed level set $\Sigma_{1}$.

Our approach is to restrict $p$ to a level set $\Sigma_{P}$, for $P>F\left(-\phi^{0}\right)$, and then show that, under the mapping $p \mapsto s p$, the quotient

$$
\left|\frac{\left.F D\left(F D^{2} F\right)\right|_{s p-\phi^{0}}(s p, \hat{q}, \hat{q})}{G(s p, s p)^{1 / 2} G(q, q)}\right|
$$

is less than $\varepsilon$ for large-enough $s$. In the above expression,

with

$$
\hat{q}=q-\frac{\left.D F\right|_{s p-\phi^{0}}(q)}{F\left(s p-\phi^{0}\right)}\left(s p-\phi^{0}\right)=q-s r(q) p+r(q) \phi^{0},
$$

$$
\lim _{s \rightarrow \infty} r(q)=0 \quad \text { and } \quad \lim _{s \rightarrow \infty} s r(q)=\left.D F\right|_{p}(q) / F(p) .
$$

As $s \rightarrow \infty$, the numerator of (8) is

$$
\begin{aligned}
\lim _{s \rightarrow \infty} & \left.F D\left(F D^{2} F\right)\right|_{s p-\phi^{0}}(s p, \hat{q}, \hat{q}) \\
\quad= & \left.\lim _{s \rightarrow \infty} F D\left(F D^{2} F\right)\right|_{p-\phi^{0} / s}\left(\phi^{0}, q-s r(q) p+r(q) \phi^{0}, q-s r(q) p+r(q) \phi^{0}\right) \\
\quad= & \left.F D\left(F D^{2} F\right)\right|_{p}\left(\phi^{0}, q-\frac{\left.D F\right|_{p}(q)}{F(p)} p, q-\frac{\left.D F\right|_{p}(q)}{F(p)} p\right)=0,
\end{aligned}
$$

by using (6).

If $q$ is not parallel to $p$, then the denominator is strictly positive:

$$
\lim _{s \rightarrow \infty} G_{s p-\phi^{0}}(s p, s p)^{1 / 2} G_{s p-\phi^{0}}(q, q) \geq \sqrt{A_{P}} G_{p}(q, q)>0,
$$

where $A_{P}$ is the constant given by Lemma 5. It follows that for each such $q$ we can find an $S$ so that $F(s p) \geq S$ implies that (8) is less than $\varepsilon$.

In the case that $q$ is parallel to $p$, without loss of generality we can set $q=p$. Multiply both numerator and denominator of (8) by $s^{2}$, so that the latter is bounded below: $\lim _{s \rightarrow \infty} G_{s p-\phi^{0}}(s p, s p)^{3 / 2} \geq A_{P}{ }^{3 / 2}>0$. The numerator is then

$$
\left.F D\left(F D^{2} F\right)\right|_{s p-\phi^{0}}\left(s p,\left(s-s^{2} r(p)\right) p+s r(p) \phi^{0},\left(s-s^{2} r(p)\right) p+s r(p) \phi^{0}\right) .
$$


Since

$$
\begin{array}{rl}
\lim _{s \rightarrow \infty} s-s^{2} & r(p) \\
& =\lim _{s \rightarrow \infty} s\left(1-\frac{\left.D F\right|_{s p-\phi^{0}}(p)}{F\left(p-\phi^{0} / s\right)}\right)=\lim _{s^{\prime} \rightarrow 0} \frac{1}{s^{\prime}}\left(\frac{\left.D F\right|_{p}(p)}{F(p)}-\frac{\left.D F\right|_{p-s^{\prime} \phi^{0}}(p)}{F\left(p-s^{\prime} \phi^{0}\right)}\right) \\
& =D\left(\frac{\left.D F\right|_{p}}{F(p)}\right)\left(\phi^{0}, p\right)=\frac{\left.D^{2} F\right|_{p}\left(\phi^{0}, p\right)}{F(p)}-\frac{\left.\left.D F\right|_{p}(p) D F\right|_{p}\left(\phi^{0}\right)}{F(p)^{2}}=0,
\end{array}
$$

the numerator approaches $\left.F D\left(F D^{2} F\right)\right|_{p}\left(\phi^{0}, \phi^{0}, \phi^{0}\right)=0$ as $s \rightarrow \infty$. That is, for $q=p$ we can find an $S$ such that, if $F(s p) \geq S$, then (8) is less that $\varepsilon$.

Since (8) is continuous in $q$, the supremum over $q \in \Sigma_{1}$ (and hence in $V^{*}$ ) of these constants $S$ is finite, and we set this to be $S_{\varepsilon}^{\prime}$. Finally, we set $S_{\varepsilon}=S_{\varepsilon}^{\prime}+F\left(-\phi^{0}\right)$, so that, whenever $F\left(p-\phi^{0}\right) \geq S_{\varepsilon}$, we have $F(p) \geq S_{\varepsilon}^{\prime}$ as well.

The next two technical lemmas are used in the proof of Theorem 3.

Lemma 8. Let $\left\{\phi^{0}, \phi^{1}, \ldots, \phi^{n}\right\}$ be a basis for $V^{*}$, where $n>1$. There are constants $k^{\prime}, k>0$ such that, for all $p=\sum_{i=1}^{n} p_{i} \phi^{i}$,

$$
k^{\prime} \leq\left.\sum_{i=1}^{n} F D^{2} F\right|_{p-\phi^{0}}\left(\phi^{i}, \phi^{i}\right) \leq k .
$$

Proof. Fix $p \in \Sigma_{1}$. Make an orthogonal change of coordinates on $\left\{\phi^{1}, \ldots, \phi^{n}\right\}$ so that $p$ is parallel to $\phi^{1}$. Note that $\left.G\right|_{p-\phi^{0}}\left(\phi^{i}, \phi^{i}\right)$ is strictly positive. Mapping $p \mapsto s p$, we notice that

$$
\begin{aligned}
\lim _{s \rightarrow \infty} & \left.G\right|_{s p-\phi^{0}}\left(\phi^{i}, \phi^{i}\right) \\
& =\left.\lim _{s \rightarrow \infty} G\right|_{s p-\phi^{0}}\left(\phi^{i}-\frac{\left.D F\right|_{s p-\phi^{0}}\left(\phi^{i}\right)}{F\left(s p-\phi^{0}\right)}\left(s p-\phi^{0}\right) \phi^{i}-\frac{\left.D F\right|_{s p-\phi^{0}}\left(\phi^{i}\right)}{F\left(s p-\phi^{0}\right)}\left(s p-\phi^{0}\right)\right) \\
& =\left.G\right|_{p}\left(\phi^{i}-\frac{\left.D F\right|_{p}\left(\phi^{i}\right)}{F(p)} p, \phi^{i}-\frac{\left.D F\right|_{p}\left(\phi^{i}\right)}{F(p)} p\right) \\
& =\left.G\right|_{\phi^{1}}\left(\phi^{i}, \phi^{i}\right),
\end{aligned}
$$

is strictly positive and finite for $i=2, \ldots, n$, as is

$$
\begin{aligned}
\left.\lim _{s \rightarrow 0} G\right|_{s p-\phi^{0}}\left(\phi^{i}, \phi^{i}\right) & =\left.G\right|_{-\phi^{0}}\left(\phi^{i}+\frac{\left.D F\right|_{-\phi^{0}}\left(\phi^{i}\right)}{F\left(-\phi^{0}\right)} \phi^{0}, \phi^{i}+\frac{\left.D F\right|_{-\phi^{0}}\left(\phi^{i}\right)}{F\left(-\phi^{0}\right)} \phi^{0}\right) \\
& =\left.G\right|_{-\phi^{0}}\left(\phi^{i}, \phi^{i}\right) .
\end{aligned}
$$

It follows that $\left.G\right|_{s p-\phi^{0}}\left(\phi^{1}, \phi^{1}\right)+\left.\sum_{i=2}^{n} G\right|_{s p-\phi^{0}}\left(\phi^{i}, \phi^{i}\right)$ has strictly positive bounds for all $s \in[0, \infty)$, and taking the minimum and maximum of these bounds over $p \in \Sigma_{1}$ gives the result. 
Lemma 9. If $F$ satisfies the symmetry condition (4), then there exists a constant $C_{2}$ depending only on $F$ such that

$$
\left.F D^{2} F\right|_{p-\phi^{0}}(p, q) \leq C_{2} \frac{F(q)}{F\left(p-\phi^{0}\right)}
$$

for all $p=\sum_{i=1}^{n} p_{i} \phi^{i}$ and $q=\sum_{i=1}^{n} q_{i} \phi^{i}$.

Proof. This property is unchanged under the mapping $q \mapsto s q$, so we may restrict $q$ to $\Sigma_{1}$. Fix such a $q$. For any given $p \in \Sigma_{1}$, consider

$$
\left.\frac{F\left(p-\phi^{0}\right)}{F(q)} F D^{2} F\right|_{p-\phi^{0}}(p, q) \text {. }
$$

Under the mapping $p \mapsto s p$ as $s \rightarrow \infty$, this becomes

$$
\begin{aligned}
& \left.\lim _{s \rightarrow \infty} \frac{F\left(s p-\phi^{0}\right)}{F(q)} F D^{2} F\right|_{s p-\phi^{0}}(s p, q) \\
& \quad=\lim _{s^{\prime} \rightarrow 0} \frac{1}{F(q)} \frac{1}{s^{\prime}}\left(\left.F\left(p-s^{\prime} \phi^{0}\right) F D^{2} F\right|_{p-s^{\prime} \phi^{0}}\left(\phi^{0}, q\right)-\left.F^{2} D^{2} F\right|_{p}\left(\phi^{0}, q\right)\right) \\
& \quad=-\left.\frac{1}{F(q)} D\left(F^{2} D^{2} F\right)\right|_{p}\left(\phi^{0}, \phi^{0}, q\right),
\end{aligned}
$$

which is bounded, as $F$ is $C^{3}$. Consequently,

$$
\left.\sup _{s \in[0, \infty)} \frac{F\left(s p-\phi^{0}\right)}{F(q)} F D^{2} F\right|_{s p-\phi^{0}}(s p, q) \leq C(p, q)<\infty
$$

for some finite $C(p, q)$. Setting $C_{2}=\max _{p, q \in \Sigma_{1}} C(p, q)$ gives the result.

\section{The gradient estimate for periodic flows}

Proof of Theorem 1. Define

$$
Z:=F\left(D u-\phi^{0}\right)-f(u, t),
$$

where $f$ is a smooth positive function for $t>0$ with

$$
f(\cdot, 0) \geq \sup _{t=0} F\left(D u-\phi^{0}\right) .
$$

Later, we will choose $f$ to be some inverse power of the fundamental solution to a heat equation (12); for now, we focus on the first part of $Z$.

Consider the first point where $Z$ is no longer negative, so that $F=f$. This point will be a spatial maximum of $Z$, owing to the periodicity of $u$. Assume that, at this point,

$$
F\left(D u-\phi^{0}\right) \geq P>F\left(-\phi^{0}\right) .
$$


The first derivative condition at this point is

$$
0=D_{k} Z=\left.D F\right|_{\nu}\left(\phi^{m}\right) u_{m k}-f^{\prime} u_{k},
$$

where $v=D u-\phi^{0}$. That is, for all vectors $v \in \operatorname{span}\left\{e_{1}, \ldots, e_{n}\right\}$,

$$
D^{2} u\left(\left.D F\right|_{v}\left(\phi^{m}\right) e_{m}, v\right)=f^{\prime} D u(v) .
$$

Using (5b), we can rewrite the evolution equation for $u$ in terms of the directions $\hat{\phi}^{i}$ tangent to the level set $\Sigma_{F(v)}$ :

$$
u_{t}=\left.F D^{2} F\right|_{\nu}\left(\phi^{i}, \phi^{j}\right) u_{i j}=\left.F D^{2} F\right|_{\nu}\left(\hat{\phi}^{i}, \hat{\phi}^{j}\right) u_{i j} .
$$

We make use of this in finding an evolution equation for $F$ :

$$
\begin{aligned}
& \frac{\partial F}{\partial t}=\left.D F\right|_{\nu}\left(\phi^{k}\right) u_{k t} \\
&=\left.D F\right|_{\nu}\left(\phi^{k}\right)\left(\left.F D^{2} F\right|_{\nu}\left(\hat{\phi}^{i}, \hat{\phi}^{j}\right) u_{i j}\right)_{k} \\
&=\left.D F\right|_{\nu}\left(\phi^{k}\right)\left(\left.D\left(F D^{2} F\right)\right|_{\nu}\left(D_{k} \nu, \hat{\phi}^{i}, \hat{\phi}^{j}\right) u_{i j}+\left.F D^{2} F\right|_{\nu}\left(D_{k} \hat{\phi}^{i}, \hat{\phi}^{j}\right) u_{i j}\right. \\
&\left.\quad+\left.F D^{2} F\right|_{\nu}\left(\hat{\phi}^{i}, D_{k} \hat{\phi}^{j}\right) u_{i j}+\left.F D^{2} F\right|_{\nu}\left(\hat{\phi}^{i}, \hat{\phi}^{j}\right) u_{i j k}\right) \\
& \quad+\left.F D^{2} F\right|_{\nu}\left(\phi^{i}, \phi^{j}\right) D_{i j} F \\
& \quad-\left.F D^{2} F\right|_{\nu}\left(\phi^{i}, \phi^{j}\right)\left(\left.D^{2} F\right|_{\nu}\left(\phi^{m}, \phi^{l}\right) u_{m i} u_{l j}+\left.D F\right|_{\nu}\left(\phi^{m}\right) u_{m i j}\right) \\
&=\left.D F\right|_{\nu}\left(\phi^{k}\right)\left(\left.D\left(F D^{2} F\right)\right|_{\nu}\left(D_{k} v, \hat{\phi}^{i}, \hat{\phi}^{j}\right) u_{i j}+\left.F D^{2} F\right|_{\nu}\left(D_{k} \hat{\phi}^{i}, \hat{\phi}^{j}\right) u_{i j}\right. \\
&\left.\quad+\left.F D^{2} F\right|_{\nu}\left(\hat{\phi}^{i}, D_{k} \hat{\phi}^{j}\right) u_{i j}\right) \\
& \quad+\left.F D^{2} F\right|_{\nu}\left(\phi^{i}, \phi^{j}\right) D_{i j} F-\left.\left.F D^{2} F\right|_{\nu}\left(\phi^{i}, \phi^{j}\right) D^{2} F\right|_{\nu}\left(\phi^{m}, \phi^{l}\right) u_{m i} u_{l j} .
\end{aligned}
$$

Here, in the third step we have added and subtracted second derivatives of $F$. The derivatives of $v$ are

$$
D_{k} v=u_{m k} \phi^{m}
$$

which we use to simplify those terms with derivatives of $\hat{\phi}^{i}$ :

$$
\begin{aligned}
\left.D^{2} F\right|_{\nu}\left(D_{k} \hat{\phi}^{i}, \hat{\phi}^{j}\right) & =\left.D^{2} F\right|_{\nu}\left(D_{k}\left(-r\left(\phi^{i}\right) v\right), \hat{\phi}^{j}\right) \\
& =\left.D^{2} F\right|_{\nu}\left(-D_{k}\left(r\left(\phi^{i}\right)\right) v-r\left(\phi^{i}\right) u_{m k} \phi^{m}, \hat{\phi}^{j}\right) \\
& =-\left.r\left(\phi^{i}\right) D^{2} F\right|_{\nu}\left(u_{m k} \phi^{m}, \hat{\phi}^{j}\right) \\
& =-\left.\frac{\left.D F\right|_{\nu}\left(\phi^{i}\right)}{F(\nu)} D^{2} F\right|_{\nu}\left(u_{m k} \phi^{m}, \hat{\phi}^{j}\right) .
\end{aligned}
$$


The evolution equation is now

$$
\begin{aligned}
\frac{\partial F}{\partial t}= & \left.\left.D F\right|_{\nu}\left(\phi^{k}\right) D\left(F D^{2} F\right)\right|_{\nu}\left(\phi^{m}, \hat{\phi}^{i}, \hat{\phi}^{j}\right) u_{m k} u_{i j} \\
& -\left.D F\right|_{\nu}\left(\phi^{k}\right)\left(\left.\left.D F\right|_{\nu}\left(\phi^{i}\right) D^{2} F\right|_{\nu}\left(\phi^{m}, \hat{\phi}^{j}\right)\right. \\
& \left.+\left.\left.D F\right|_{\nu}\left(\phi^{j}\right) D^{2} F\right|_{\nu}\left(\phi^{m}, \hat{\phi}^{i}\right)\right) u_{m k} u_{i j} \\
& +\left.F D^{2} F\right|_{\nu}\left(\phi^{i}, \phi^{j}\right) D_{i j} F-\left.\left.F D^{2} F\right|_{\nu}\left(\phi^{i}, \phi^{j}\right) D^{2} F\right|_{\nu}\left(\phi^{m}, \phi^{l}\right) u_{m i} u_{l j}
\end{aligned}
$$

At a critical point of $Z$, we can use the first-derivative condition (9) to simplify further. The first term of (10) becomes

$$
\begin{aligned}
\left.D^{2} u\left(\left.D F\right|_{\nu}\left(\phi^{k}\right) e_{k}, e_{m}\right) D\left(F D^{2} F\right)\right|_{\nu} & \left(\phi^{m}, \hat{\phi}^{i}, \hat{\phi}^{j}\right) u_{i j} \\
& =\left.f^{\prime} D u\left(e_{m}\right) D\left(F D^{2} F\right)\right|_{\nu}\left(\phi^{m}, \hat{\phi}^{i}, \hat{\phi}^{j}\right) u_{i j} \\
& =\left.f^{\prime} D\left(F D^{2} F\right)\right|_{\nu}\left(D u, \hat{\phi}^{i}, \hat{\phi}^{j}\right) u_{i j},
\end{aligned}
$$

while the second becomes

$$
\begin{aligned}
-\left.\left.\left.D F\right|_{\nu}\left(\phi^{k}\right) D F\right|_{\nu}\left(\phi^{i}\right) D^{2} F\right|_{\nu}\left(\phi^{m}, \hat{\phi}^{j}\right) u_{m k} u_{i j} \\
\quad \times\left. D^{2} F\right|_{\nu}\left(\phi^{m}, \hat{\phi}^{j}\right) D^{2} u\left(\left.D F\right|_{\nu}\left(\phi^{k}\right) e_{k}, e_{m}\right) D^{2} u\left(\left.D F\right|_{\nu}\left(\phi^{i}\right) e_{i}, e_{j}\right) \\
=-\left.f^{\prime 2} D^{2} F\right|_{\nu}\left(D u\left(e_{m}\right) \phi^{m}, D u\left(e_{j}\right) \hat{\phi}^{j}\right) \\
=-\left.f^{\prime 2} D^{2} F\right|_{\nu}(D u, D u),
\end{aligned}
$$

as does the third. Thus, the evolution equation is

$$
\begin{aligned}
\frac{\partial F}{\partial t}=\frac{f^{\prime}}{f} & \left.F D\left(F D^{2} F\right)\right|_{v}\left(D u, \hat{\phi}^{i}, \hat{\phi}^{j}\right) u_{i j}-\left.2 \frac{f^{\prime 2}}{f} F D^{2} F\right|_{\nu}(D u, D u) \\
& +\left.F D^{2} F\right|_{\nu}\left(\phi^{i}, \phi^{j}\right) D_{i j} F-\left.\left.\frac{1}{f} F D^{2} F\right|_{\nu}\left(\phi^{i}, \phi^{j}\right) F D^{2} F\right|_{\nu}\left(\phi^{m}, \phi^{l}\right) u_{m i} u_{l j},
\end{aligned}
$$

where we have multiplied some terms through by $1=F / f$ in order that derivatives of $F$ appear as homogeneous degree-zero terms.

Derivatives of $f$ are given by

$$
D f=f^{\prime} D u, \quad D_{i j} f=f^{\prime \prime} u_{i} u_{j}+f^{\prime} u_{i j}, \quad \frac{d f}{d t}=f^{\prime} u_{t}+f_{t},
$$

for $i, j \neq 0$, so an evolution equation for $f$ is

$$
\begin{aligned}
\frac{d f}{d t} & =f^{\prime} u_{t}+f_{t}+\left.F D^{2} F\right|_{\nu}\left(\phi^{i}, \phi^{j}\right)\left(D_{i j} f-f^{\prime \prime} u_{i} u_{j}-f^{\prime} u_{i j}\right) \\
& =f_{t}+\left.F D^{2} F\right|_{v}\left(\phi^{i}, \phi^{j}\right)\left(D_{i j} f-f^{\prime \prime} u_{i} u_{j}\right) .
\end{aligned}
$$


The entire evolution equation for $Z$, at a local maximum, is

$$
\begin{aligned}
\frac{d Z}{d t} & =\left.F D^{2} F\right|_{\nu}\left(\phi^{i}, \phi^{j}\right) D_{i j} Z+\left.\frac{f^{\prime}}{f} F D\left(F D^{2} F\right)\right|_{\nu}\left(D u, \hat{\phi}^{i}, \hat{\phi}^{j}\right) u_{i j} \\
& -\left.2 \frac{f^{\prime 2}}{f} F D^{2} F\right|_{\nu}(D u, D u)-\left.\left.\frac{1}{f} F D^{2} F\right|_{\nu}\left(\phi^{i}, \phi^{j}\right) F D^{2} F\right|_{\nu}\left(\phi^{m}, \phi^{l}\right) u_{m i} u_{l j} \\
& -f_{t}+\left.F D^{2} F\right|_{\nu}(D u, D u) f^{\prime \prime} .
\end{aligned}
$$

Notice that all the covectors $\phi^{i}$ and $D u$ appear in places where replacing them by their projections in the tangent space of $\Sigma_{F(v)}$ (that is, by $\hat{\phi}^{i}$ or $\widehat{D u}$ ) has no effect, thanks to (5b) and (5c). On the tangent space, $D^{2} F$ is positive definite. Choose the basis $\left\{\phi^{1}, \ldots, \phi^{n}\right\}$ so that $G_{v}$ is the identity at the maximum point, $G_{\nu}{ }^{\alpha \beta}=\delta^{\alpha \beta}$. The evolution equation for $Z$ is now

$$
\begin{aligned}
\frac{d Z}{d t}=G^{i j} D_{i j} Z & +\left.\frac{f^{\prime}}{f} F D\left(F D^{2} F\right)\right|_{\nu}\left(\widehat{D u}, \hat{\phi}^{i}, \hat{\phi}^{j}\right) u_{i j} \\
& -2 \frac{f^{\prime 2}}{f} G(D u, D u)-\frac{1}{f} G^{i j} G^{m l} u_{m i} u_{l j}-f_{t}+G(D u, D u) f^{\prime \prime} .
\end{aligned}
$$

The Cauchy-Schwarz inequality for a positive-definite matrix $B$ implies that $v^{T} w \leq \varepsilon v^{T} B v+(4 \varepsilon)^{-1} w^{T} B^{-1} w$. We use this to estimate the second term of (11):

$$
\begin{aligned}
\left.\frac{f^{\prime}}{f} F D\left(F D^{2} F\right)\right|_{\nu} & \left(\widehat{D u}, \hat{\phi}^{i}, \hat{\phi}^{j}\right) u_{i j} \\
= & \frac{f^{\prime}}{f}\left(\left.\left.D F\right|_{\nu}(\widehat{D u}) F D^{2} F\right|_{\nu}\left(\hat{\phi}^{i}, \hat{\phi}^{j}\right)+\left.F^{2} D^{3} F\right|_{\nu}\left(\widehat{D u}, \hat{\phi}^{i}, \hat{\phi}^{j}\right)\right) u_{i j} \\
= & \frac{f^{\prime}}{f} u_{k} Q^{k i j} u_{i j} \leq \varepsilon \frac{f^{\prime 2}}{f} G(D u, D u)+\frac{1}{4 \varepsilon f} G_{\alpha \beta} Q^{\alpha i j} u_{i j} Q^{\beta k l} u_{k l},
\end{aligned}
$$

where the first term of the second line is zero because $\widehat{D u}$ is tangent to the unit ball, so $\left.D F\right|_{\nu}(\widehat{D u})=0$. In the last line, we have used the notation $G_{\alpha \beta}=\left(G^{-1}\right)^{\alpha \beta}$ for the inverse.

We can use condition (3), on the smallness of third derivatives, to estimate the second term in this inequality:

$$
\begin{aligned}
& \frac{1}{4 \varepsilon f} G_{\alpha \beta} Q^{\alpha i j} u_{i j} Q^{\beta k l} u_{k l} \\
& =\frac{1}{4 \varepsilon f} Q\left(G_{\alpha \beta} \hat{\phi}^{\alpha}, u_{i j} \hat{\phi}^{i}, \hat{\phi}^{j}\right) Q\left(\hat{\phi}^{\beta}, u_{k l} \hat{\phi}^{k}, \hat{\phi}^{l}\right) \\
& \leq \frac{C_{1}^{2}}{4 \varepsilon f}\left(G\left(G_{\alpha \beta} \hat{\phi}^{\alpha}, G_{\gamma \beta} \hat{\phi}^{\gamma}\right) G\left(u_{i j} \hat{\phi}^{i}, u_{m j} \hat{\phi}^{m}\right) G\left(\hat{\phi}^{j}, \hat{\phi}^{j}\right)\right. \\
& \left.\times G\left(\hat{\phi}^{\beta}, \hat{\phi}^{\beta}\right) G\left(u_{k l} \hat{\phi}^{k}, u_{p l} \hat{\phi}^{p}\right) G\left(\hat{\phi}^{l}, \hat{\phi}^{l}\right)\right)^{1 / 2}
\end{aligned}
$$




$$
=\frac{C_{1}^{2}}{4 \varepsilon f}\left(G_{\beta \beta} u_{i j} u_{i j} G^{j j} G^{\beta \beta} u_{k l} u_{k l} G^{l l}\right)^{1 / 2}=\frac{C_{1}^{2}}{4 \varepsilon f} \sqrt{n}\left(G^{i j} G^{k l} u_{i k} u_{j l}\right) .
$$

Now we can estimate (11) from above:

$$
\begin{aligned}
\frac{d Z}{d t} \leq G^{i j} D_{i j} Z+\frac{1}{f}\left(\frac{C_{1}^{2}}{4 \varepsilon} \sqrt{n}-1\right) & G^{i j} G^{k l} u_{i k} u_{j l} \\
& +\frac{f^{\prime 2}}{f}(\varepsilon-2) G(D u, D u)-f_{t}+f^{\prime \prime} G(D u, D u) .
\end{aligned}
$$

The second term is zero if we choose $\varepsilon=C_{1}^{2} \sqrt{n} / 4<1$.

Choose $f=\Phi^{-q}$ for some $q>1$ and with

$$
\Phi(u, t)=\frac{1}{\sqrt{t}} \exp \left(-A \frac{(u-2 M)^{2}}{4 t}\right),
$$

which satisfies the heat equation $\Phi_{t}=A \Phi^{\prime \prime}$, where $A=A_{P}$ is the constant given by Lemma 5. Since

$$
\begin{aligned}
& f^{\prime}=-q \Phi^{-q-1} \Phi^{\prime}, \\
& f^{\prime \prime}=q(q+1) \Phi^{-q-2}\left(\Phi^{\prime}\right)^{2}-q \Phi^{-q-1} \Phi^{\prime \prime}, \\
& f_{t}=-q \Phi^{-q-1} \Phi_{t},
\end{aligned}
$$

the equation satisfied by $f$ is

$$
f_{t}=A f^{\prime \prime}-A\left(1+q^{-1}\right) f^{\prime 2} / f
$$

If we substitute $\Phi$ and its derivatives for $f$ and its derivatives, we find that

$$
\begin{aligned}
& \frac{f^{\prime 2}}{f}(\varepsilon-2) G(D u, D u)-f_{t}+f^{\prime \prime} G(D u, D u) \\
& =q^{2} \Phi^{-q-2} \Phi^{\prime 2}(\varepsilon-2) G(D u, D u) \\
& \quad+\left(q(q+1) \Phi^{-q-2} \Phi^{\prime 2}-q \Phi^{-q-1} \Phi^{\prime \prime}\right) G(D u, D u)+A q \Phi^{-q-1} \Phi^{\prime \prime} \\
& =q \Phi^{-q-2} \Phi^{\prime 2}(q(\varepsilon-1)+1) G(D u, D u)+q \Phi^{-q-1} \Phi^{\prime \prime}(A-G(D u, D u)) .
\end{aligned}
$$

The first term is zero if we choose $q^{-1}=1-\varepsilon=1-C_{1}^{2} \sqrt{n} / 4$.

As we assumed at the beginning that $F\left(D u-\phi^{0}\right) \geq P$, Lemma 5 implies that $G(D u, D u) \geq A_{P}$. As $\Phi^{\prime \prime}$ is positive for small times, for $t<T^{\prime}$ we have $\partial Z / \partial t \leq 0$.

On the other hand, if we consider the possibility that $F\left(D u-\phi^{0}\right)=f<P$ at this local maximum, we could replace $f$ by $\sup \{f, P\}$ in the definition of $Z$. In that case, the first maximum of $Z$ occurs at a point where the barrier is flat, and so the first variation is

$$
0=D_{k} Z=\left.D F\right|_{z}\left(\phi^{k}\right) u_{m k}
$$


and the evolution equation for $Z$ at the local maximum is

$$
\frac{d Z}{d t}=\left.F D^{2} F\right|_{v}\left(\phi^{i}, \phi^{j}\right) D_{i j} Z-\left.\left.D^{2} F\right|_{\nu}\left(\phi^{i}, \phi^{j}\right) F D^{2} F\right|_{\nu}\left(\phi^{m}, \phi^{l}\right) u_{m i} u_{l j} \leq 0 .
$$

Since $Z_{t} \leq 0$ at the first point where $Z=0$, we have $Z \leq 0$ for all $t<T^{\prime}$. The same argument works if, in the definition (12) of $\Phi$, the term $u-2 M$ is replaced by $u+2 M$. The conclusion (with $|u|-2 M$ ) follows.

Proof of Theorem 2. We begin by defining $Z$ as in Theorem 1, and assume that the first non-negative value of $Z$ occurs when $F\left(D u-\phi^{0}\right) \geq S_{\varepsilon}$, for some $\varepsilon>0$ to be chosen later and with the corresponding $S_{\varepsilon}$ given by Lemma 7 . We then follow the earlier proof up to equation (11), the evolution equation for $Z$ at a local maximum.

This time, we choose the local coordinates $\left\{\phi^{1}, \ldots, \phi^{n}\right\}$ so that at this point $D^{2} u$ is diagonal. This puts the second term of (11) in a suitable form to be estimated using Lemma 7.

$$
\begin{aligned}
& \left.\frac{f^{\prime}}{f} F D\left(F D^{2} F\right)\right|_{\nu}\left(D u, \hat{\phi}^{j}, \hat{\phi}^{j}\right) u_{j j} \\
& \quad \leq\left|\varepsilon \frac{f^{\prime}}{f} \sqrt{G(D u, D u)} G\left(\hat{\phi}^{j}, \hat{\phi}^{j}\right) u_{j j}\right| \leq \frac{f^{\prime 2}}{2 f} G(D u, D u)+\frac{\varepsilon^{2}}{2 f}\left|G\left(\hat{\phi}^{j}, \hat{\phi}^{j}\right) u_{i j}\right|^{2} \\
& \quad \leq \frac{f^{\prime 2}}{2 f} G(D u, D u)+\frac{\varepsilon^{2}}{2 f} n G^{i j} u_{j k} G^{k l} u_{l i},
\end{aligned}
$$

where in the last line we used the trace inequality $(\operatorname{trace} A)^{2} \leq n \operatorname{trace}\left(A^{2}\right)$.

If we now choose $\varepsilon=\sqrt{2 / n}$, the second term of this inequality is cancelled by the fourth term of (11). The evolution equation becomes

$$
\frac{d Z}{d t} \leq G^{i j} D_{i j} Z-\frac{3}{2} \frac{f^{\prime 2}}{f} G(D u, D u)-f_{t}+G(D u, D u) f^{\prime \prime}
$$

This is negative at a local maximum if we make the same choice of barrier as before, $f=\Phi^{-q}$ for $q=2$, $\Phi$ given by (12), and $A=A_{S_{\varepsilon}}$ given by Lemma 5 .

If our assumption that $F\left(D u-\phi^{0}\right) \geq S_{\varepsilon}$ does not hold, then we can replace $f$ by $\max \left\{S_{\varepsilon}, f\right\}$. At the local maximum, $Z_{t} \leq 0$. The conclusion follows.

Remark. In the last theorem, we have chosen $q=2$ somewhat arbitrarily; in fact $q$ needs only be strictly greater than 1 , since we can set $q=\left(1-n \varepsilon^{2} / 4\right)^{-1}$, for $\varepsilon$ given by Lemma 7 . However, a smaller $\varepsilon$ may force a larger $S_{\varepsilon}$, so the optimal choice would depend on the exact form of $F$. 


\section{Interior estimate for anisotropic mean curvature flow}

Proof of Theorem 3. We introduce a localizing term $\eta$ into our definition of $Z$,

$$
Z:=F\left(D u-\phi^{0}\right)-\frac{f}{\eta},
$$

which is now restricted to the shrinking ball

$$
(x, t) \in B_{\sqrt{R^{2}-2 k t}} \times[0, T],
$$

where $k$ is the constant given by Lemma 8 .

The smooth strictly positive function $f=f(u, t)$ is chosen so that $Z<0$ at the initial time, while $\eta$ is a smooth positive function chosen so that $\eta \rightarrow 0$ on the boundary of the shrinking ball.

Assume that, at the first interior point where $Z=0$,

$$
F\left(D u-\phi^{0}\right) \geq P>F\left(-\phi^{0}\right) .
$$

Then $F(D u)=f / \eta$ and, as this is a spatial maximum (since the choice of $\eta$ ensures that there are no boundary maxima), we have a first derivative condition

$$
0=D_{k} Z=\left.D F\right|_{\nu}\left(\phi^{m}\right) u_{m k}-D_{k}(f / \eta) .
$$

An evolution equation for $f / \eta$, with second derivatives $G^{i j} D_{i j}(f / \eta)$ added and subtracted, follows:

$$
\begin{array}{r}
\frac{d}{d t}\left(\frac{f}{\eta}\right)=\frac{1}{\eta}\left(f^{\prime} u_{t}+f_{t}\right)-\frac{f}{\eta^{2}} \frac{d \eta}{d t}+\left.F D^{2} F\right|_{\nu}\left(\phi^{i}, \phi^{j}\right) D_{i j}\left(\frac{f}{\eta}\right) \\
-\left.F D^{2} F\right|_{\nu}\left(\phi^{i}, \phi^{j}\right)\left(\frac{1}{\eta}\left(f^{\prime \prime} u_{i} u_{j}+f^{\prime} u_{i j}\right)-\frac{f^{\prime}}{\eta^{2}}\left(u_{j} D^{i} \eta+u_{i} D^{j} \eta\right)\right. \\
\left.+2 \frac{f}{\eta^{3}} D^{i} \eta D^{j} \eta-\frac{f}{\eta^{2}} D_{i j} \eta\right) \\
=G^{i j} D_{i j}\left(\frac{f}{\eta}\right)+\frac{1}{\eta}\left(f_{t}-G(D u, D u) f^{\prime \prime}\right)-\frac{f}{\eta^{2}}\left(\frac{d}{d t}-G^{i j} D_{i j}\right) \eta \\
+2 \frac{f^{\prime}}{\eta^{2}} G(D u, D \eta)-2 \frac{f}{\eta^{3}} G(D \eta, D \eta) .
\end{array}
$$

We can incorporate the first derivative condition (13) into (10), the evolution equation for $F$ :

$$
\begin{aligned}
\frac{d F}{d t}= & G^{i j} D_{i j} F+\left.D_{m}(f / \eta) D\left(F D^{2} F\right)\right|_{\nu}\left(\phi^{m}, \hat{\phi}^{i}, \hat{\phi}^{j}\right) u_{i j} \\
& -\left.2 F D^{2} F\right|_{\nu}(D(f / \eta), D(f / \eta))-\left.\left.F D^{2} F\right|_{\nu}\left(\phi^{i}, \phi^{j}\right) D^{2} F\right|_{\nu}\left(\phi^{m}, \phi^{l}\right) u_{m i} u_{l j}
\end{aligned}
$$




$$
\begin{aligned}
=G^{i j} & D_{i j} F+\left.\frac{\eta}{f} F D\left(F D^{2} F\right)\right|_{\nu}\left(D(f / \eta), \hat{\phi}^{i}, \hat{\phi}^{j}\right) u_{i j} \\
& -2 \frac{\eta}{f}\left(\frac{f^{\prime 2}}{\eta^{2}} G(D u, D u)-2 \frac{f f^{\prime}}{\eta^{3}} G(D u, D \eta)+\frac{f^{2}}{\eta^{4}} G(D \eta, D \eta)\right) \\
& -\frac{\eta}{f} G^{i j} G^{m l} u_{m i} u_{l j} .
\end{aligned}
$$

Putting the last two steps together gives an evolution equation for $Z$ at a local maximum:

$$
\begin{aligned}
\frac{d Z}{d t}=G^{i j} & D_{i j} Z+\left.\frac{\eta}{f} F D\left(F D^{2} F\right)\right|_{\nu}\left(D(f / \eta), \hat{\phi}^{i}, \hat{\phi}^{j}\right) u_{i j} \\
& -\frac{\eta}{f} G^{i j} G^{m l} u_{m i} u_{l j}-\frac{1}{\eta}\left(f_{t}-G(D u, D u) f^{\prime \prime}+2 \frac{f^{\prime 2}}{f} G(D u, D u)\right) \\
& +\frac{f}{\eta^{2}}\left(\frac{d}{d t}-G^{i j} D_{i j}\right) \eta+2 \frac{f^{\prime}}{\eta^{2}} G(D u, D \eta) .
\end{aligned}
$$

The second term here may be split into a part with $D f$ and a part with $D \eta$ :

$$
\begin{aligned}
& \left.\frac{\eta}{f} F D\left(F D^{2} F\right)\right|_{v}\left(\frac{f^{\prime}}{\eta} D u-\frac{f}{\eta^{2}} D \eta, \hat{\phi}^{i}, \hat{\phi}^{j}\right) u_{i j} \\
& =\left.\frac{f^{\prime}}{f} F D\left(F D^{2} F\right)\right|_{v}\left(D u, \hat{\phi}^{i}, \hat{\phi}^{j}\right) u_{i j}-\left.\frac{1}{\eta} F D\left(F D^{2} F\right)\right|_{v}\left(D \eta, \hat{\phi}^{i}, \hat{\phi}^{j}\right) u_{i j}
\end{aligned}
$$

These may be individually estimated using the Cauchy-Schwarz inequality and the condition on smallness of third derivatives, as described in the proof of Theorem 1:

$$
\begin{aligned}
& \left.\frac{f^{\prime}}{f} F D\left(F D^{2} F\right)\right|_{\nu}\left(D u, \hat{\phi}^{i}, \hat{\phi}^{j}\right) u_{i j} \\
& \leq \mu_{1} \frac{f^{\prime 2}}{f \eta} G(D u, D u)+\frac{1}{4 \mu_{1}} \frac{\eta}{f} C_{1}^{2} \sqrt{n}\left(G^{i j} G^{m l} u_{m i} u_{l j}\right) \\
& -\left.\frac{1}{\eta} F D\left(F D^{2} F\right)\right|_{\nu}\left(D \eta, \hat{\phi}^{i}, \hat{\phi}^{j}\right) u_{i j} \\
& \quad \leq \mu_{2} \frac{f}{\eta^{3}} G(D \eta, D \eta)+\frac{1}{4 \mu_{2}} \frac{\eta}{f} C_{1}^{2} \sqrt{n}\left(G^{i j} G^{k l} u_{i k} u_{j l}\right)
\end{aligned}
$$

for some $0<\mu_{1}, \mu_{2}<1$.

We choose the localising term $\eta:=\tilde{\eta}^{r}$ for some $r>1$ and $\tilde{\eta}=R^{2}-2 k t-|x|^{2}$. Then

$$
\begin{aligned}
D_{i} \eta & =r \tilde{\eta}^{r-1} D_{i} \tilde{\eta}, \\
D_{i j} \eta & =r \tilde{\eta}^{r-1} D_{i j} \tilde{\eta}+r(r-1) \tilde{\eta}^{r-2} D_{i} \tilde{\eta} D_{j} \tilde{\eta}
\end{aligned}
$$


and the second-to-last term of the evolution equation (14) is

$$
\begin{aligned}
\frac{f}{\eta^{2}}\left(\frac{d}{d t}-G^{i j} D_{i j}\right) \eta & =\frac{f}{\eta^{2}} r \tilde{\eta}^{r-1}\left(-2 k+2 \operatorname{trace} G-(r-1) \tilde{\eta}^{-1} G(D \tilde{\eta}, D \tilde{\eta})\right) \\
& \leq \frac{f}{\eta^{2}} r \tilde{\eta}^{r-2}(1-r) G(D \tilde{\eta}, D \tilde{\eta}) .
\end{aligned}
$$

As $F$ satisfies the symmetry condition (4), we may use Lemma 9 to estimate the final term of the evolution equation:

$$
\begin{aligned}
2 \frac{f^{\prime}}{\eta^{2}} G(D u, D \eta) & =\left.2 \frac{f^{\prime}}{\eta^{2}} F D^{2} F\right|_{D u-\phi^{0}}(D u, D \eta) \\
& \leq 2 \frac{f^{\prime}}{\eta^{2}} \frac{C_{2} F(D \eta)}{F\left(D u-\phi^{0}\right)}=2 C_{2} F(D \eta) \frac{f^{\prime}}{f \eta} \leq 2 C_{2} C_{3} r R^{2 r-1} \frac{f^{\prime}}{f \eta}
\end{aligned}
$$

where we have used that $F(D \eta)=r \tilde{\eta}^{r-1} F(D \tilde{\eta}) \leq C_{3} r R^{2 r-1}$, for a $C_{3}>0$ depending only on $F$.

The evolution equation can now be estimated from above:

$$
\begin{aligned}
& \frac{d Z}{d t} \leq G^{i j} D_{i j} Z+\frac{\eta}{f}\left(\frac{1}{4 \mu_{1}} C_{1}^{2} \sqrt{n}+\right.\left.\frac{1}{4 \mu_{2}} C_{1}^{2} \sqrt{n}-1\right) G^{i j} G^{m l} u_{m i} u_{l j} \\
&-\frac{1}{\eta}\left(f_{t}-G(D u, D u) f^{\prime \prime}+\left(2-\mu_{1}\right) \frac{f^{\prime 2}}{f} G(D u, D u)-2 C_{2} F(D \eta) \frac{f^{\prime}}{f}\right) \\
&+\frac{f}{\eta^{2}} r \tilde{\eta}^{r-2}\left(1-r+r \mu_{2}\right) G(D \tilde{\eta}, D \tilde{\eta}) .
\end{aligned}
$$

Since $C_{1}^{2} \sqrt{n} / 4<1 / 2$, we can choose $\mu_{1}<1$ and $\mu_{2}<1$ such that

$$
\frac{C_{1}^{2} \sqrt{n}}{4}\left(\frac{1}{\mu_{1}}+\frac{1}{\mu_{2}}\right) \leq 1
$$

With such choices, the second term of the evolution inequality (16) will be negative. We can also set $r=\left(1-\mu_{2}\right)^{-1}>1$, so the coefficient of $\tilde{\eta}^{-1} G(D \tilde{\eta}, D \tilde{\eta})$ is zero.

As in the previous cases, we set $f=\Phi^{-q}$ with $\Phi$ given by (12) for $A=A_{P}$ given by Lemma 5 . The part in parentheses of the second line of (16) is then

$$
\begin{aligned}
&-\frac{1}{\eta}\left(f_{t}-\right.\left.G(D u, D u) f^{\prime \prime}+\left(2-\mu_{1}\right) \frac{f^{\prime 2}}{f} G(D u, D u)-2 C_{2} F(D \eta) \frac{f^{\prime}}{f}\right) \\
&= \frac{1}{\eta}\left(q \Phi^{-q-1} \Phi_{t}+G(D u, D u)\left(q(q+1) \Phi^{-q-2} \Phi^{\prime 2}-q \Phi^{-q-1} \Phi^{\prime \prime}\right)\right. \\
&\left.-\left(2-\mu_{1}\right) G(D u, D u) q^{2} \Phi^{-q-2} \Phi^{\prime 2}+2 C_{1} C_{2} r R^{2 r-1} \Phi^{-1}\left|\Phi^{\prime}\right|\right)
\end{aligned}
$$




$$
\begin{aligned}
=\frac{q \Phi^{-q-1}}{\eta} & \left(\Phi_{t}-G(D u, D u) \Phi^{\prime \prime}\right) \\
& +\frac{q\left|\Phi^{\prime}\right|}{\eta \Phi}\left(G(D u, D u)\left|\Phi^{\prime}\right| \Phi^{-q-1}\left(1-q+q \mu_{1}\right)+2 C_{1} C_{2} r R^{2 r-1}\right) .
\end{aligned}
$$

If we choose $T^{\prime}$ small enough that $\Phi^{\prime \prime} \geq 0$, then the term

$$
\Phi_{t}-G(D u, D u) \Phi^{\prime \prime}=(A-G(D u, D u)) \Phi^{\prime \prime}
$$

is negative. Additionally, if we choose $T^{\prime}$ small enough that $\Phi \leq 1$, then

$$
G(D u, D u)\left|\Phi^{\prime}\right| \Phi^{-q-1} \geq \frac{A^{2}}{t} M \Phi^{-q} \geq \frac{A^{2} M}{T^{\prime}},
$$

and so, for the last term to be negative, we need only choose $q$ large enough that

$$
q \geq \frac{1}{1-\mu_{1}}\left(1+\frac{2 C_{1} C_{2} r R^{2 r-1} T^{\prime}}{A^{2} M}\right) .
$$

So, at such maxima, $Z_{t} \leq 0$.

At local maxima where $F\left(D u-\phi^{0}\right)<P$, we replace $f / \eta$ in the definition of $Z$ by $\max \{f / \eta, P\}$, in which case the barrier is flat at the local maxima, and we again find that $Z_{t} \leq 0$.

In either case, the maximum principle ensures that $Z$ is never greater than zero, and the conclusion follows.

\section{References}

[Andrews 2001] B. Andrews, "Volume-preserving anisotropic mean curvature flow", Indiana Univ. Math. J. 50:2 (2001), 783-827. MR 2002m:53105 Zbl 1047.53037

[Andrews and Clutterbuck 2005] B. Andrews and J. Clutterbuck, "Time-interior gradient estimates for quasilinear parabolic equations", preprint, 2005.

[Bao et al. 2000] D. Bao, S.-S. Chern, and Z. Shen, An introduction to Riemann-Finsler geometry, vol. 200, Graduate Texts in Mathematics, Springer-Verlag, New York, 2000. MR 2001g:53130 Zbl 0954.53001

[Barles et al. 2002] G. Barles, S. Biton, and O. Ley, "A geometrical approach to the study of unbounded solutions of quasilinear parabolic equations", Arch. Ration. Mech. Anal. 162:4 (2002), 287-325. MR 2003b:35091 Zbl 1052.35084

[Colding and Minicozzi 2004] T. H. Colding and W. P. Minicozzi, II, "Sharp estimates for mean curvature flow of graphs", J. Reine Angew. Mathematik 574 (2004), 187-195. MR 2005g:53119 Zbl 1057.53050

[Ecker and Huisken 1989] K. Ecker and G. Huisken, "Mean curvature evolution of entire graphs", Ann. of Math. (2) 130:3 (1989), 453-471. MR 91c:53006 Zbl 0696.53036

[Ecker and Huisken 1991] K. Ecker and G. Huisken, "Interior estimates for hypersurfaces moving by mean curvature", Invent. Math. 105:3 (1991), 547-569. MR 92i:53010 Zbl 0707.53008

[Evans and Spruck 1992] L. C. Evans and J. Spruck, "Motion of level sets by mean curvature, III", J. Geom. Anal. 2:2 (1992), 121-150. MR 93d:58044 Zbl 0768.53003 
[Winklmann 2005] S. Winklmann, "Estimates for stable hypersurfaces of prescribed $F$-mean curvature”, Manuscripta Math. 118:4 (2005), 485-499. MR 2006k:53106 Zbl 05000076

Received March 15, 2005.

Julie ClutTERBUCK

Mathematical Sciences Institute

Australian National University

ACT 0200

Australia

Julie.Clutterbuck@maths.anu.edu.au 